\title{
Identification of Diverse Adenosine-to-Inosine RNA Editing Subtypes in Colorectal Cancer
}

\author{
Si-Hyun Lee, $\mathrm{MS}^{1}$ \\ Hwang-Phill Kim, $\mathrm{PhD}^{1,2}$ \\ Jun-Kyu Kang, MS1,2 \\ Sang-Hyun Song, $\mathrm{PhD}^{2}$ \\ Sae-Won Han, MD, PhD1,3 \\ Tae-You Kim, MD, PhD',2,3
}

${ }^{1}$ Department of Molecular Medicine and Biopharmaceutical Sciences, Graduate School of Convergence Science and Technology, Seoul National University College of Medicine, Seoul, ${ }^{2}$ Cancer Research Institute, Seoul National University College of Medicine, Seoul, ${ }^{3}$ Department of Internal Medicine, Seoul National University Hospital, Seoul, Korea

Correspondence: Tae-You Kim, MD, PhD Department of Internal Medicine, Seoul National University College of Medicine, 101 Daehak-ro, Jongno-gu, Seoul 03080, Korea

Tel: $82-2-2072-7200$

Fax: 82-2-762-9662

E-mail: kimty@snu.ac.kr

Received July 12, 2016

Accepted January 12, 2017

Published Online January 25, 2017

*Si-Hyun Lee and Hwang-Phill Kim contributed equally to this work.

\section{Purpose}

RNA editing generates protein diversity by altering RNA sequences in coding regions without changing the overall DNA sequence. Adenosine-to-inosine (A-to-I) RNA editing events have recently been reported in some types of cancer, but they are rare in human colorectal cancer (CRC). Therefore, this study was conducted to identify diverse RNA editing in CRC.

\section{Materials and Methods}

We compared transcriptome data of $39 \mathrm{CRC}$ samples and paired adjacent tissues from The Cancer Genome Atlas database to identify RNA editing patterns in CRC, focusing on canonical A-to-I RNA edits in coding sequence regions. We investigated nonsynonymous RNA editing patterns by comparing tumor and normal tissue transcriptome data.

\section{Results}

The number of RNA edits varied from 12 to 42 per sample. We also observed that hypoand hyper-RNA editing patterns were distinguishable within the samples. We found 10 recurrent nonsynonymous RNA editing candidates in nine genes (PDLIM, NEIL1, SRP9, GLI1, APMAP, IGFBP7, ZNF358, COPA, and ZNF587B) and validated some by Sanger sequencing and the inosine chemical erasing assay. We further showed that editing at these positions was performed by the adenosine deaminase acting on RNA 1 enzyme. Most of these genes are hypoedited in CRC, but editing of GLI1 was increased in cancer tissues compared with normal tissues.

\section{Conclusion}

Our results show that nonsynonymous RNA editing patterns can be used to identify CRC patients and could serve as novel biomarkers for CRC.

\section{Introduction}

Genomic alterations are a major cause of cancer initiation and progression [1,2]; however, they do not account for all of the changes in gene expression that are observed in cancer. RNA editing is a post-transcriptional molecular modification process that changes specific nucleotide sequences within an mRNA molecule after it has been generated by RNA polymerase. Therefore, RNA editing contributes to the diversity

\section{Key words}

RNA editing, Colorectal neoplasms, Transcriptome sequencing, GLI family zinc finger 1 , Adenosine deaminase 
by reverse transcription and translation machinery [5].

The recent development of next generation sequencing technologies has caused the number of identified RNA edits to increase dramatically. Several groups have been studying new methods to detect RNA editing [6,7], leading to identification of several million potential RNA editing sites in the human genome $[8,9]$. Most of these sites are in noncoding and repetitive elements regions, whereas RNA editing in coding regions is rare [10]. However, these editing events are very important because they can cause amino acid changes that lead to functional alterations of the encoded proteins.

These alterations may be responsible for activating oncogenes or inactivating tumor suppressors, thus affecting tumor progression. Several studies have reported a hypoediting pattern in glioblastoma (GBM) [11,12]. In GBM, unedited $G l u R-B$ enhanced malignancy [13], whereas A-to-I editing of AZIN1, which was increased in hepatocellular carcinoma specimens, resulted in amino acid changes that promoted cell proliferation [14]. We previously reported A-to-I editing of the Ras homologue family member $R H O Q$ and demonstrated that edited $R H O Q$ was associated with increased invasiveness [15]. These results show the importance of RNA editing in cancer, but few studies have investigated RNA editing sites in colorectal cancer (CRC).

In this study, we focused on finding nonsynonymous RNA editing sites in CRC using RNA sequencing data from The Cancer Genome Atlas (TCGA) database [2]. To accomplish this, we analyzed 39 paired colorectal tumors and adjacent normal tissue samples to identify recurrent editing sites that could be used as biomarkers for detection of CRC.

\section{Materials and Methods}

\section{Nonsynonymous A-to-I RNA editing calling pipeline}

We acquired RNA sequencing data from the TCGA database (https://tcga-data.nci.nih.gov) [2]. Bam files were analyzed using VarScan2 [16]. We compared data from normal and tumor samples with the following variant calling parameters: min-coverage, 8 ; min-var-freq, 0.2; mina-avg-qual, 20; and min-reads, 2. Variant call files were annotated with ANNOVAR [17]. We selected the positions that were converted from $A$ to $G$, nonsynonymous single nucleotide variants (nsSNVs), after filtering somatic mutations that were enrolled in TCGA. We also removed all of the data from dbsnp137, excluding cDNA data and the positions within similar genomic regions.

\section{Primary human colorectal tissues}

To compare GLI1 RNA editing between tumor and normal tissue samples, 10 human colorectal tumor tissues and the matched normal tissues were obtained from the tissue bank of Seoul National University Hospital (Seoul, Korea).

\section{DNA and RNA isolation and cDNA synthesis}

Genomic DNA (gDNA) from cell lines was isolated using the QIAMP DNA mini kit (Qiagen, Valencia, CA) according to the manufacturer's instructions. Total RNA was extracted from each cell line using an RNeasy mini kit (Qiagen) according to the manufacturer's instructions, after which $1 \mu \mathrm{g}$ of total RNA was used to synthesize cDNA with random hexamers and ImProm-II reverse transcriptase (Promega, Madison, WI).

\section{Polymerase chain reaction and Sanger sequencing analy- sis}

RNA editing sites were amplified by polymerase chain reaction (PCR) using target-specific primers in gDNA or cDNA templates. Information regarding the primer is described in S1 Table. Purified PCR products were sequenced with a BigDye Terminator v3.1 cycle sequencing kit (Applied Biosystems, Carlsbad, CA), then analyzed with a 3730 ABI capillary electrophoresis system (Applied Biosystems).

\section{Pyro-sequencing analyses of GLI1 RNA editing}

We designed GLI1 target-specific primers for pyrosequencing analysis. The reverse primer was biotin-labeled. Singlestranded biotinylated PCR products were processed for pyrosequencing analysis according to the manufacturer's standard protocols (PyroMark Q96 ID, Qiagen). The primer sequences were as follows: forward, 5 '-GACCGTCCTGCTCCAGCTAG-3'; reverse, 5'-CCCAACTTCTGGCTCTTCCTGT-3'; sequencing, 5'-AATGCTGCCATGGAT-3'.

\section{Inosine chemical erasing assay}

The inosine chemical erasing (ICE) assay is composed of four steps: (1) RNA cyanoethylation, (2) cDNA synthesis by reverse transcription, (3) PCR amplification, and (4) direct sequencing. We cyanoethylated total RNA from cancer cells containing edited transcripts for 15 minutes at $70^{\circ} \mathrm{C}$ alongside controls lacking cyanoethylation. The ICE assay was performed as previously described [18]. 


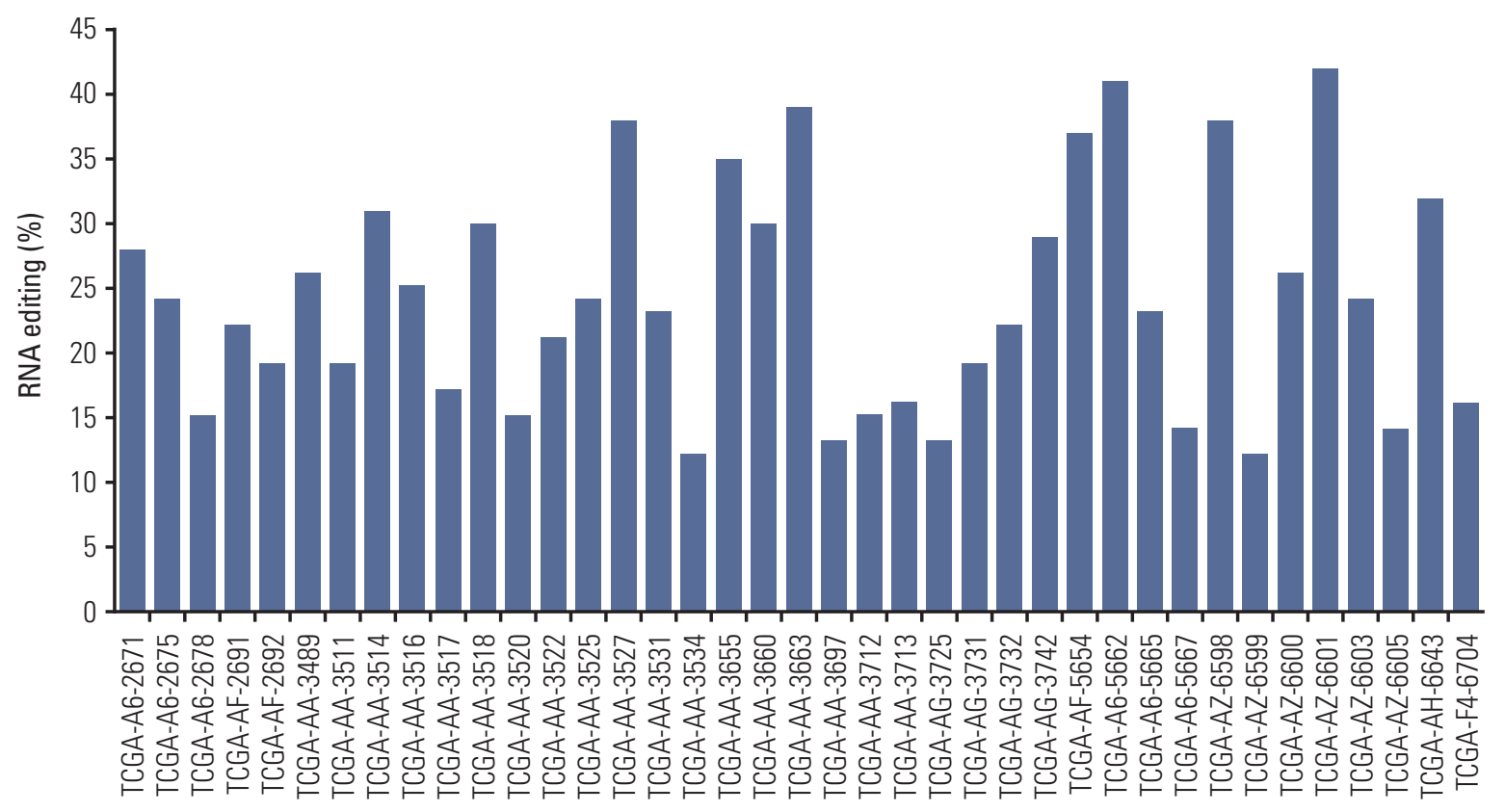

Patient

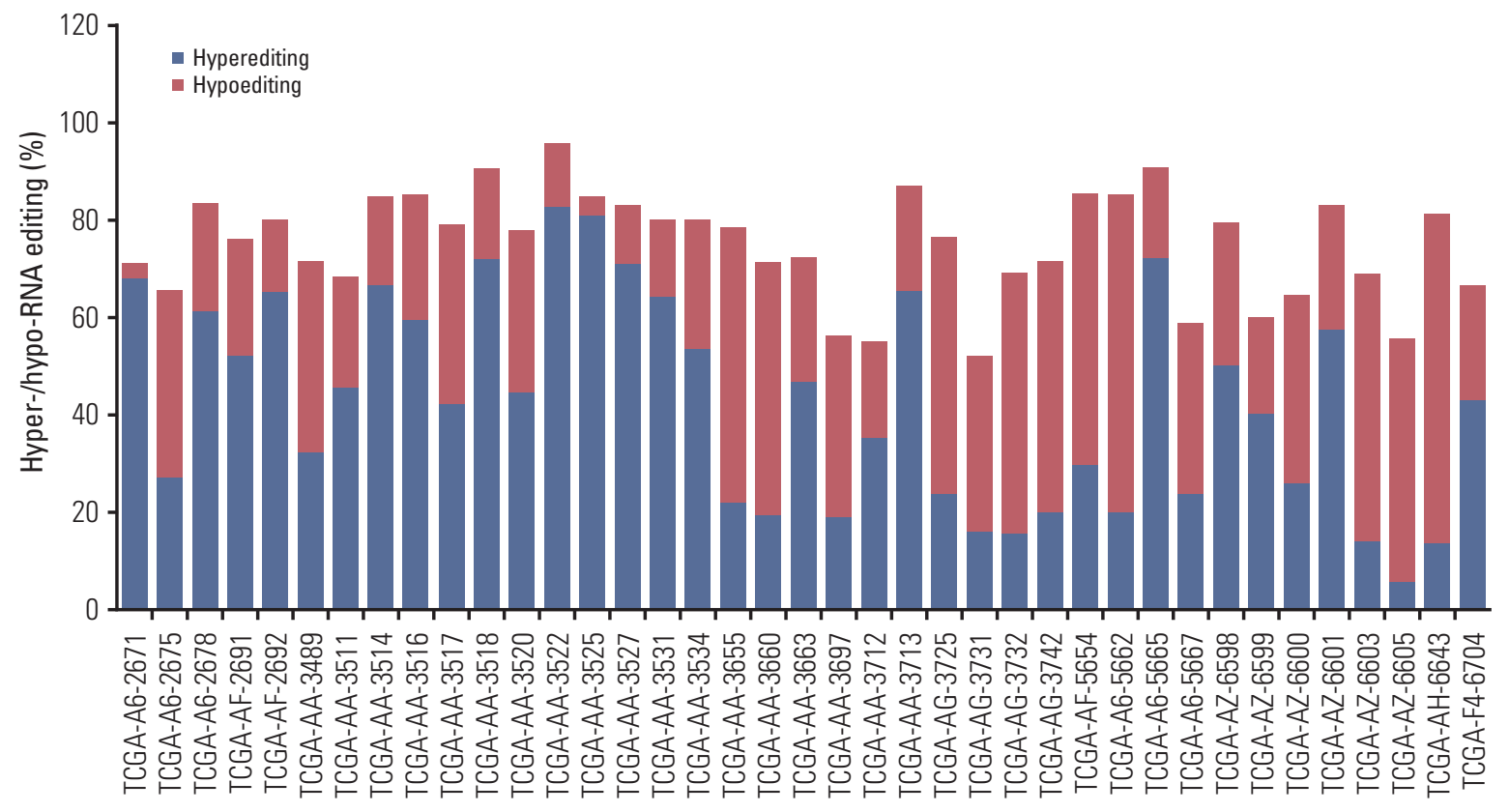

Patient

Fig. 1. The pattern of nonsynonymous RNA editing. (A) Overall number of nonsynonymous A-to-I RNA editing sites in each sample. The number of samples was 39. (B) Percentage of hyper- and hypo-RNA editing. Hyperediting was defined as editing in more than $10 \%$ in tumor than normal tissue. Hypoediting was defined as editing of less than 10\% in tumor than normal tissue. 


\section{Cell culture and siRNA transfection}

SNU-254 and SNU-81 human CRC cell lines were obtained from the Korea Cell Line Bank [19]. The cell line was grown in RPMI-1640 with $10 \%$ fetal bovine serum and gentamicin $(10 \mu \mathrm{g} / \mathrm{mL})$ at $37^{\circ} \mathrm{C}$ in a $5 \% \mathrm{CO}_{2}$-humidified atmosphere. siRNA was purchased from Mbiotech Inc. (Seoul, Korea). A total of $20 \mathrm{nM}$ siRNA was applied to cells in culture using G-fectin (Genolution, Seoul, Korea) according to manufacturer's standard protocols.

\section{Western blotting}

Cultured cells were washed with ice-cold phosphatebuffered saline and lysed with lysis buffer $(50 \mathrm{mM}$ Tris- $\mathrm{HCl}$, $\mathrm{pH} 7.5,1 \% \mathrm{NP}-40,0.1 \%$ sodium deoxycholate, $150 \mathrm{mM} \mathrm{NaCl}$, $50 \mathrm{mM} \mathrm{NaF}, 1 \mathrm{mM}$ sodium pyrophosphate, $1 \mathrm{mM}$ EDTA, and protease/phosphatase inhibitors). Lysates were transferred to new eppendorf tubes after centrifugation at 13,000 rpm for 20 minutes. Protein concentrations were quantified with a Bicinchoninic Acid Protein Assay Reagent (Pierce, Rockford, IL) according to manufacturer's instructions. Samples containing equal quantities of total proteins were resolved on sodium dodecyl sulfate-polyacrylamide denaturing gel, then transferred to nitrocellulose membranes. The membranes were subsequently incubated in blocking solution containing $1 \%$ skim milk and $1 \%$ bovine serum albumin for 1 hour at room temperature, then probed overnight at $4^{\circ} \mathrm{C}$ with primary antibodies (anti-adenosine deaminase acting on RNA 1 [ADAR1], Abcam, Cambridg, MA; anti-actin, Santa Cruz Biotechnology, Santa Cruz, CA).

\section{Statistics}

The statistical significance of the results was calculated by unpaired Student's tests, with p-values of $<0.05$ considered to be significant.

\section{Results}

\section{The pattern of RNA editing in nonsynonymous editing sites}

We downloaded 39 paired tumor and nontumor RNA transcriptome bam files from the TCGA database to analyze RNA editing patterns. The amount of total reads can influence the number of editing sites [20]; therefore, we evaluated only those samples with similar reads between normal and tumor tissues to reduce bias related to total reads. We utilized strict filtering conditions to find RNA editing candidates, using the VarScan2 software to identify RNA editing sites. VarScan2 was originally developed to identify somatic mutations and copy number alterations in exome sequencing data, but it can also be applied to analyze RNA-seq data. We set the analysis standards as follows: total reads, more than 8; editing frequency, 20; quality score, 20; and edited reads, 3 . We then only selected nonsynonymous A to $G$ conversions based on changes in AA in the refGene column, which was generated by ANNOVAR. We removed the positions enrolled in dbsnp137 except from those that originated from cDNA, similar genomic regions, and somatic mutations from the TCGA database (S2 Fig.). Overall, we identified 939 editing sites from 39 paired samples, including recurrent positions, ranging from 12 to 42 sites in each sample, with an

Table 1. List of recurrent nonsynonymous RNA editing sites

\begin{tabular}{rlllcc}
$\begin{array}{l}\text { No. of } \\
\text { detected }\end{array}$ & Gene & \multicolumn{1}{c}{ Position } & Amino acid change & $\begin{array}{c}\text { No. of hyperedited } \\
\text { samples }\end{array}$ & $\begin{array}{c}\text { No. of hypoedited } \\
\text { samples }\end{array}$ \\
\hline 18 & PDLIM & chr10:97023630 & c.A524G:p.N175S & 6 & 3 \\
18 & NEIL1 & chr15:75646086 & c.A725G:p.K242R & 7 & 7 \\
30 & SRP9 & chr1:225974614 & A192G:p.I64M & 5 & 9 \\
\hline 5 & GLI1 & chr12:57864624 & c.A2101G:p.R701G & 3 & 0 \\
\hline 6 & APMAP & chr20:24964558 & c.A193G:p.I65V & 2 & 2 \\
\hline 36 & IGFBP7 & chr4:57976234 & c.A284G:p.K95R & 0 & 33 \\
\hline 5 & IGFBP7 & chr4:57976286 & A232G:p.R78G & 0 & 5 \\
\hline 2 & ZNF587B & chr19:58355689 & A1188G:p.I396M & 0 & 2 \\
\hline 4 & COPA & chr1:160302244 & A490G:p.I164V & 0 & 4 \\
\hline 2 & ZNF358 & chr19:7585273 & c.A1145G:p.K382R & 0 & 2 \\
\hline
\end{tabular}



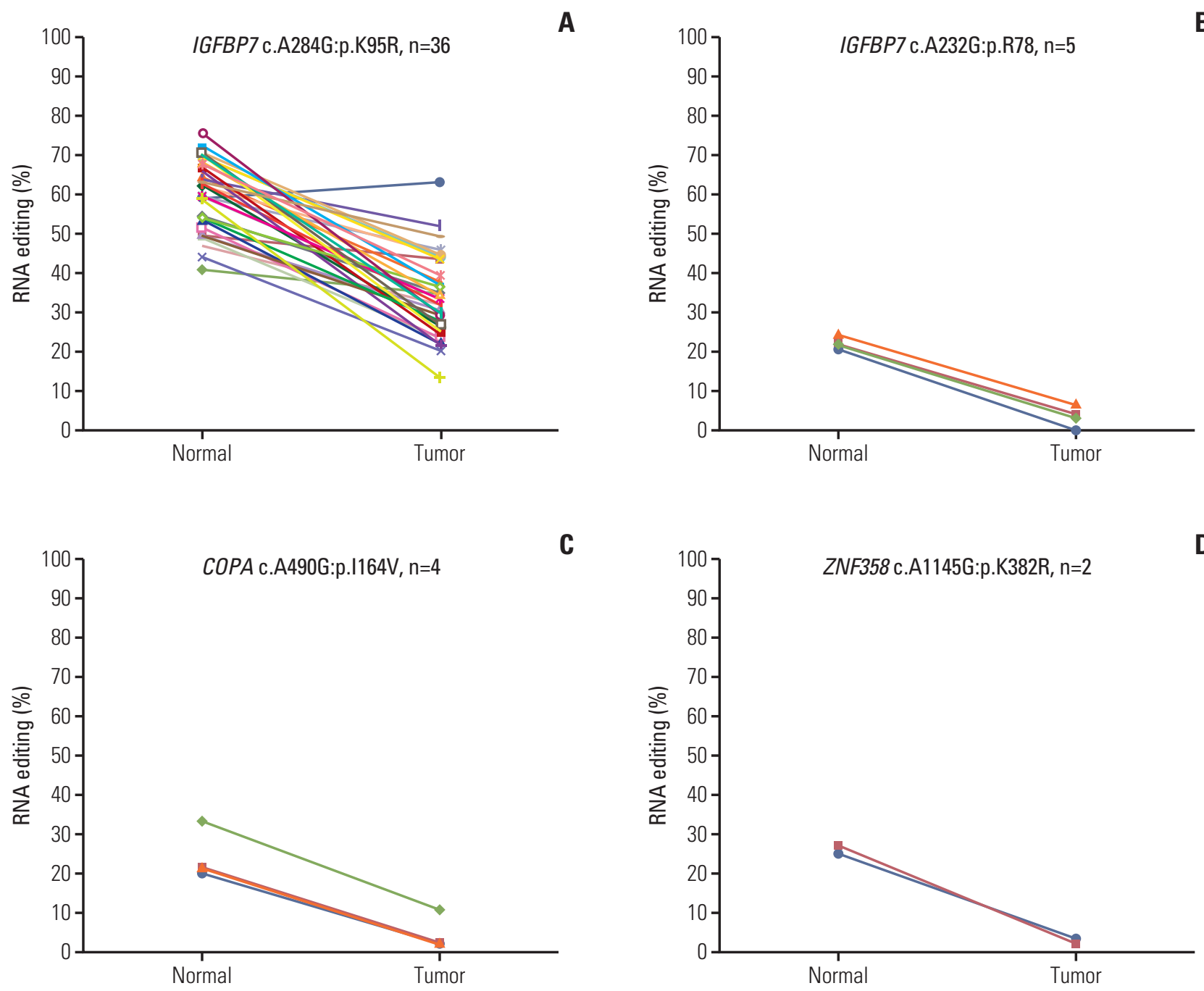

Fig. 2. Hypoediting sites in colorectal cancer. RNA editing detected among 39 paired specimens of colorectal tumor and adjacent normal tissues. The percentage of RNA editing was obtained from the The Cancer Genome Atlas transcriptome dataset. The p-value was calculated by a Student's t test. (A, B) IGFBP7 (chr4:57976234, c.A284G, p.K95R) and (chr4:57976286, c.A232G, p.R78). (C) COPA (chr1:160302244, c.A490G, p.I164V). (D) ZNF358 (chr19:7585273, c.A1145G, p.K382R).

average of 24 (Fig. 1A). We also investigated the percentage of hypo- and hyper-RNA editing within the samples. We defined hyperediting as a situation in which the percentage of RNA editing was $10 \%$ greater in tumors than adjacent normal tissue. There was no trend in hypoediting or hyperediting within the samples investigated. Among the 39 samples, 26 were classified as hyperedited and 13 were hypoedited (Fig. 1B).

We then focused on the recurrent hyper- or hypo-RNA editing sites in CRC. First, we listed the positions that were detected in more than two samples. The total number of recurrent detected editing positions was 39. Although we used relatively strict filtering standards, the possibility of false-positives remained; therefore, we employed additional filtering steps using the IGV AND PLAST programs. After these rounds of filtering, we found 10 recurrent editing positions in nine genes (Table 1 ).

\section{The hypoediting sites in CRC}

To identify RNA editing biomarkers for CRC, we investigated RNA editing by dividing edited messages into two categories: hypoediting, when the level of RNA editing is higher in normal than tumor samples; and hyperediting, when the 

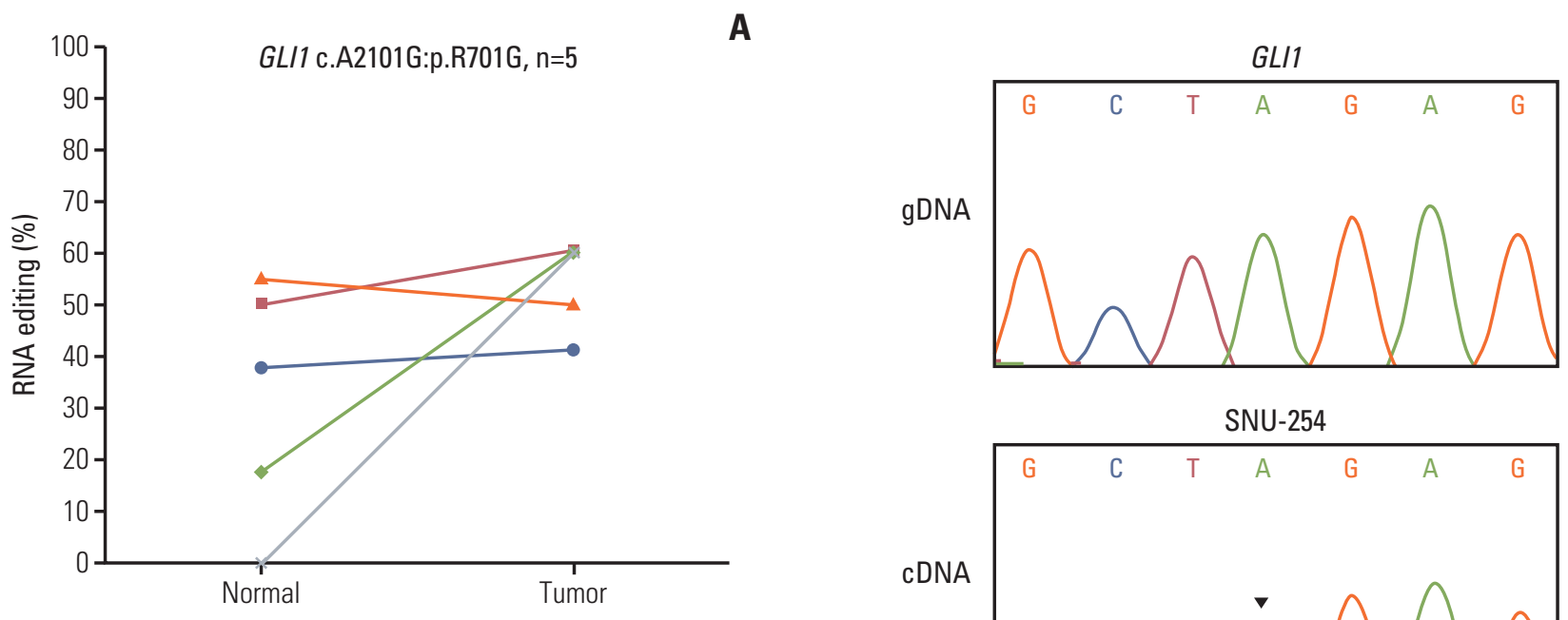

B

A
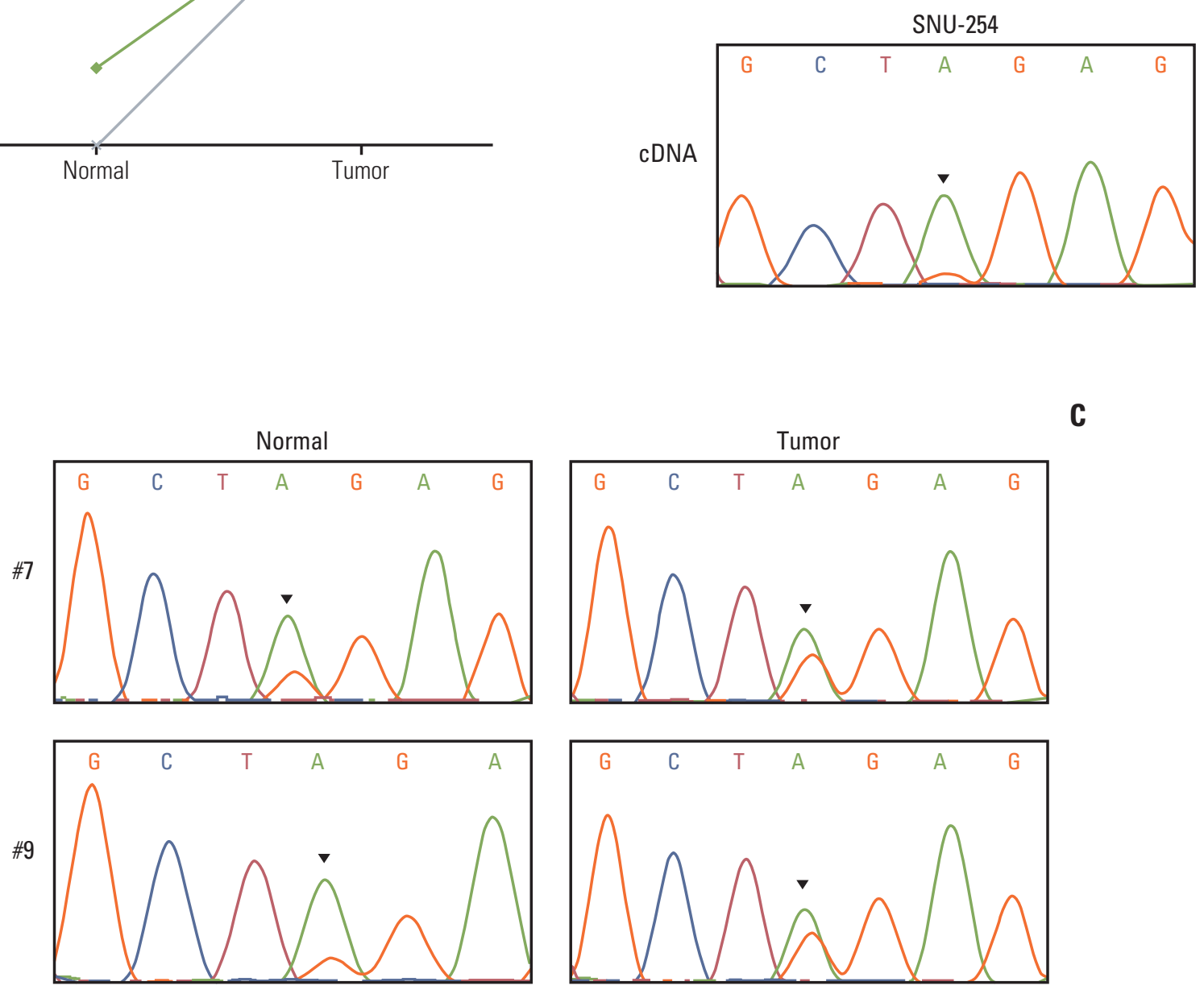

Fig. 3. RNA editing of GLI1 is hyperedited in colorectal cancer. (A) GLI1 RNA editing (chr12:57864624, c.A2101G:p.R701G) detected in The Cancer Genome Atlas transcriptome data. (B) GLI1 RNA editing validated in gDNA (top) and cDNA (bottom) by Sanger sequencing in SNU-254 cells. (C) cDNA of normal and tumor tissue was amplified, and sequencing was performed by Sanger sequencing. (Continued to the next page)

level of RNA editing is higher in tumor samples. The results revealed four positions in three genes that were hypoedited in cancer. Hypo-RNA editing of IGFBP7 (c.A284G, p.K95R) was detected in 33 samples. The other hypo-RNA editing position of IGFBP7 (c.A232G, p.R78G) was detected in five samples (Fig. 2A and B), COPA, Coatomer Protein Complex, Subunit Alpha, was detected in four samples (Fig. 2C), and ZNF358, Zinc Finger Protein 358, was detected in two samples (Fig. 2D). These messages were significantly less frequently edited in cancer. Moreover, PDLIM, NEIL1, SRP9, 


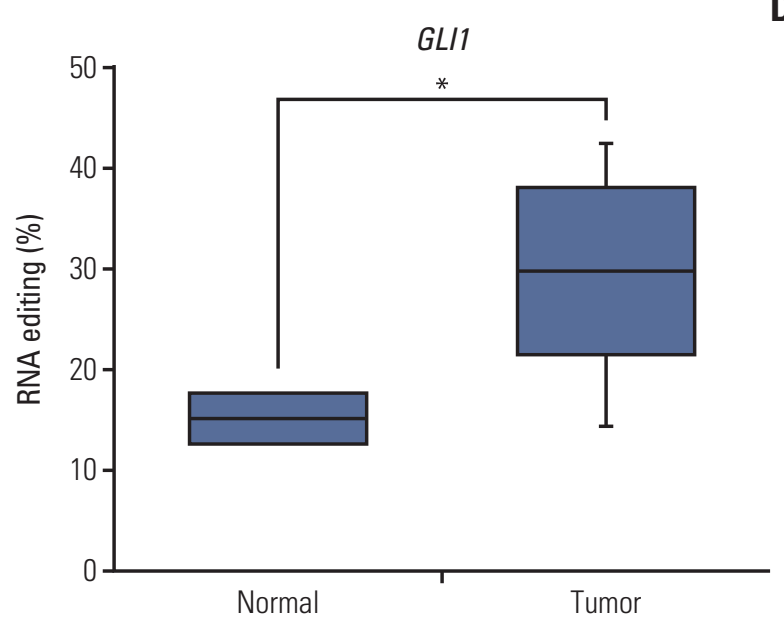

Fig. 3. (Continued from the previous page) (D) GLI1 editing percentage; the percentage of editing was determined using pyrosequencing. $n=10$; the $p$-value was calculated by a Student's t test ( $\left.{ }^{*} \mathrm{p}<0.05\right)$.

Table 2. Results of pyrosequencing in 10 colorectal tumor and normal tissues

\begin{tabular}{|ccc|}
\hline Sample No. & Normal ED (\%) & Tumor ED (\%) \\
\hline 1 & 12.7 & 42.3 \\
\hline 2 & 28 & 29.3 \\
\hline 3 & 15.9 & 33.6 \\
\hline 4 & ND & 42.1 \\
\hline 5 & ND & 29.5 \\
\hline 6 & 3.3 & ND \\
\hline 7 & 17.5 & 26.9 \\
\hline 8 & 17.4 & 14.1 \\
\hline 9 & 13.9 & 29.8 \\
\hline 10 & 12.3 & 15.7 \\
\hline Average & 15.1 & 29.3 \\
\hline
\end{tabular}

ED, RNA editing; ND, not detected.

and $A P M A P$ had both hyper- and hypo-RNA editing in 39 samples.

\section{RNA editing of GLI1 is hyperedited in CRC}

GLI1 RNA editing was detected in five samples, and was increased in colon cancer compared with normal tissue (Fig. 3A). We validated this position using Sanger sequencing (Fig. 3A) based on the pyrosequencing results of CRC cell lines (S3 Fig.). The guanosine peak was detected in cDNA, but not gDNA. After identifying GLI1 701G nsSNV, we confirmed the hyperedited pattern in CRC using Sanger sequencing in our tissue samples (Fig. 3C). There were increased guanosine peaks in tumor samples. To quantitatively analyze A-to-I RNA editing of GLI1, we conducted pyrosequencing in 10 paired samples of CRC and normal samples to measure the edited cDNA sequence. The extent of RNA editing of GLI1 was $29.3 \%$ in tumor tissues, while it was only $15.1 \%$ in normal tissues (Table 2, Fig. 3D). When compared with normal tissues, the level of RNA editing was increased by about 2-fold in tumor tissues. We also analyzed the clinical significance of GLI1 RNA editing in a TCGA cohort ( $\mathrm{n}=39)$. More GLI1 was expressed in GLI1 RNA editing samples than in samples without GLI1 RNA editing (S4 Fig.). High expression or GLI1 RNA editing showed a significant relationship with the clinical prognosis (S5 Fig.). The clinical significance of GLI1 RNA editing merits further investigation in a larger patient population. Moreover, we found that the age, sex, primary site, histology, stage, microsatellite stable/microsatellite instable status, and KRAS mutation was not significantly related to GLI1 RNA editing (S6 Table). These data suggest that GLI1 is hyperedited in CRC.

\section{Validation of the editing candidates}

To confirm the reliability of our RNA editing discovery methods, we validated editing events using Sanger sequencing of gDNA and cDNA from the CRC cell lines. The cell lines that contain RNA editing were selected based on our RNA sequencing data. RNA editing of SRP9 and NEIL1 was validated because they were also detected in our cell line RNA sequencing data. Sanger sequencing confirmed the presence of a G peak in the cDNA of SRP9 and NEIL1 (Fig. 4A). The ICE assay is another method to validate A-to-I RNA editing, as cyanoethylated inosine will not be converted to guanosine by reverse transcriptase. We conducted an ICE assay to validate RNA editing of SRP9 and NEIL1. From these experiments, we found that the guanosine peaks were significantly decreased in the treated versus the nontreated samples in both positions (Fig. 4B). These data confirm the existence of A-to-I RNA editing from our analysis and, therefore, exclude the possibility of gDNA contamination.

ADAR family enzymes mediate A-to-I RNA editing. To investigate whether ADAR enzymes were involved in the RNA editing sites we discovered in CRC, we conducted RNAi-mediated ADAR knockdown. The expression of ADAR was reduced to approximately $40 \%$ of the wild type mRNA and protein levels (Fig. 4D). We next performed Sanger sequencing to determine the ratio of $\mathrm{A} / \mathrm{G}$. The editing level was reduced in both SRP9 and NEIL1 editing positions (G: 

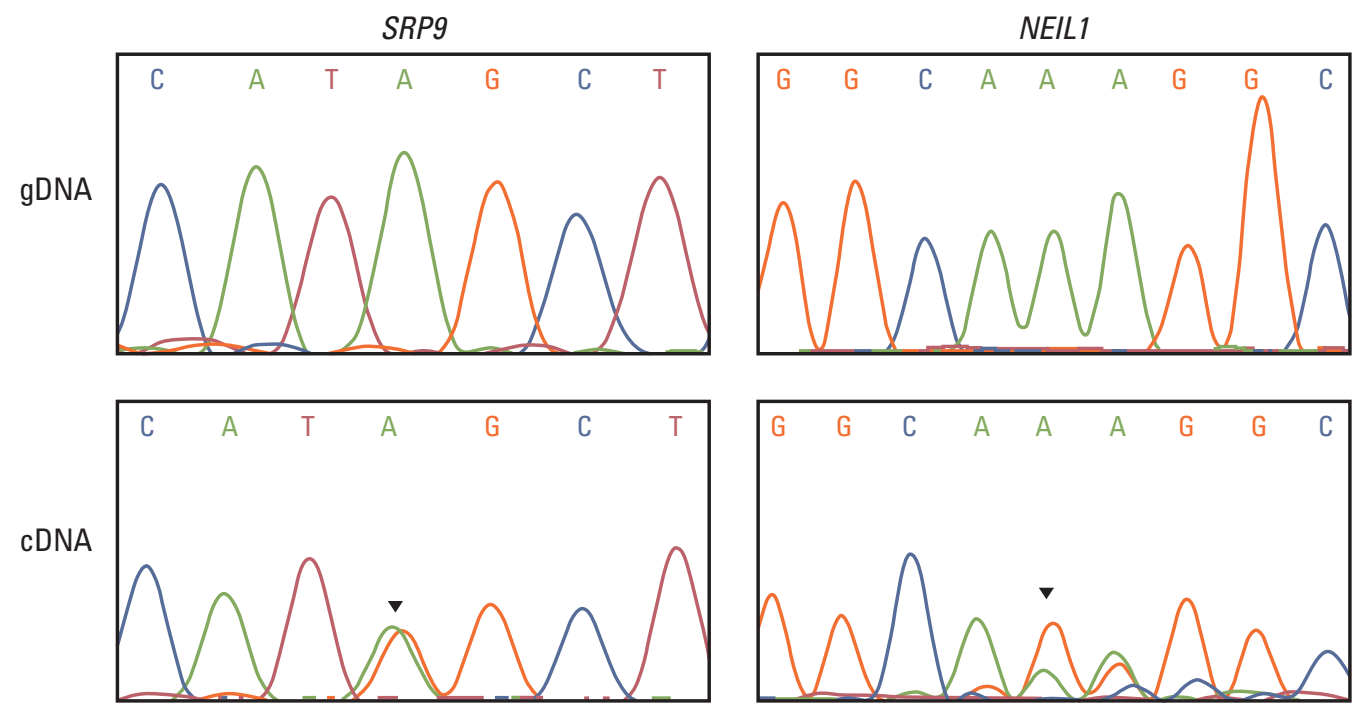

B
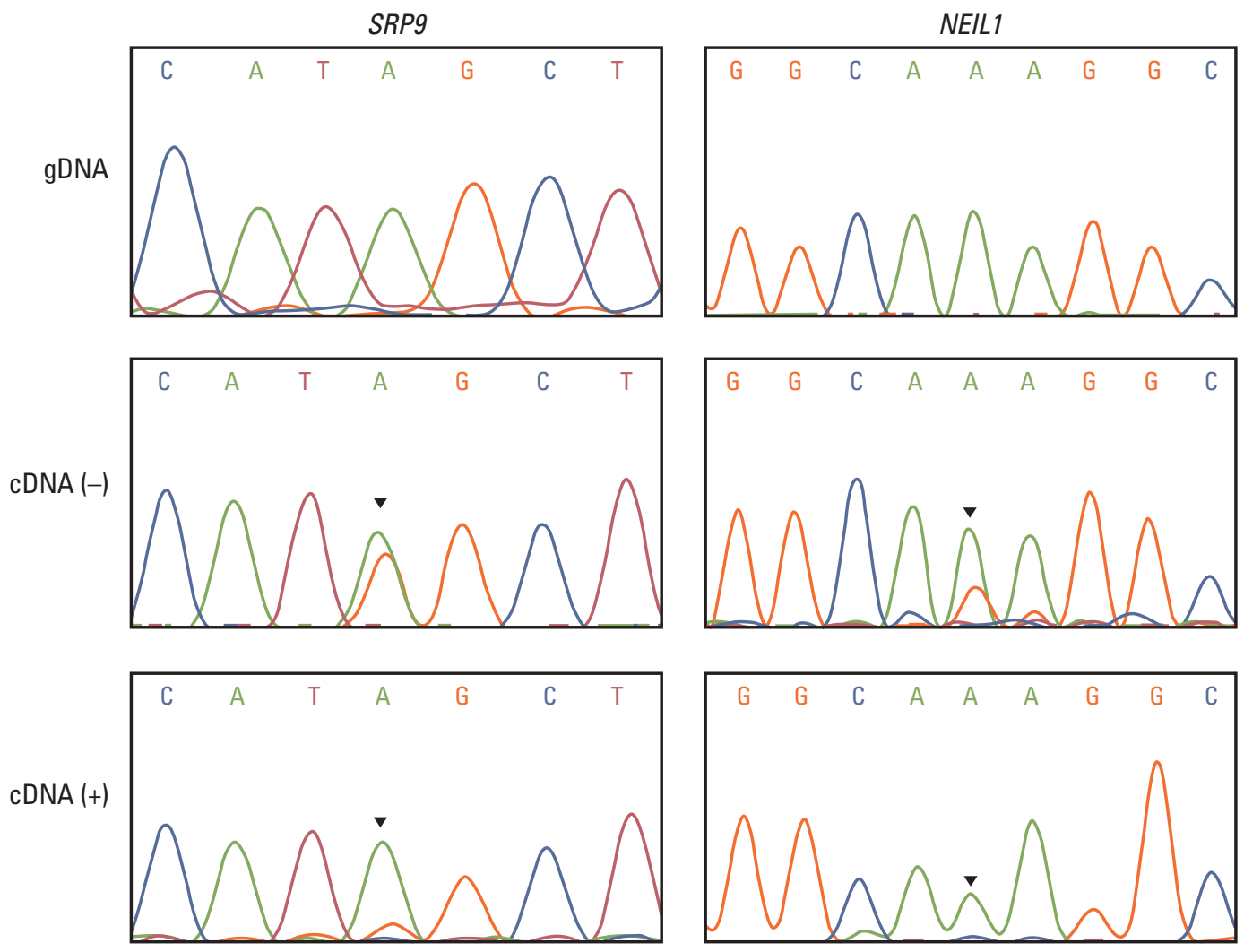

Fig. 4. Validation of the editing candidates. (A) RNA editing of SRP9 (chr1:225974614) and NEIL1 (chr15:75646086) was validated in gDNA (top) and cDNA (bottom) by Sanger sequencing in SNU-81 cells. (B) The top panels show the chromatograms of regions amplified from gDNA in SNU-81 cells. The middle panels show the chromatograms of cDNA amplified from nontreated cyanoethylation. Cyanoethylation treated RNA was amplified and their chromatograms are displayed along the bottom line. (Continued to the next page) 

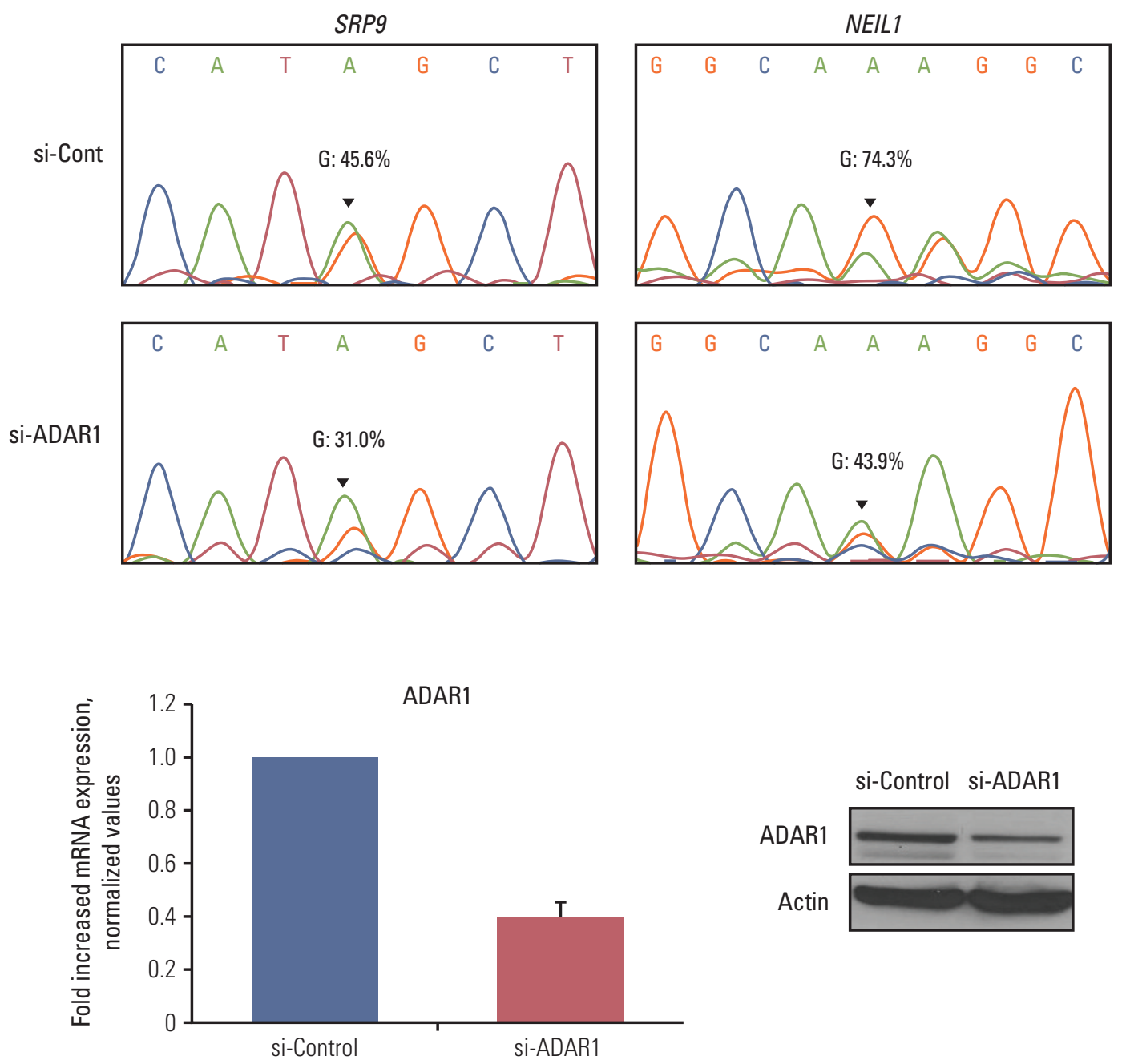

D

Fig. 4. (Continued from the previous page) (C) Sanger sequencing results of SRP9 and NEIL1 from siRNA control (top) and adenosine deaminase acting on RNA 1 (ADAR1; bottom) treated SNU-81 cells. (D) The siRNA knockdown efficiency of ADAR1 was tested by real-time quantitative reverse transcription-polymerase chain reaction (left) and western blot (right).

$45.6 \% \rightarrow 31.0 \%$ in SRP9, G:74.3\% $\rightarrow 43.9 \%$ in NEIL1) in si-RNA ADAR treated cells (Fig. 4C). Although there were limitations to these samples, the results show that the identified RNA editing candidates exist, and are affected by ADAR enzyme knockdown.

\section{Discussion}

The development of high-throughput techniques has accelerated the identification of RNA editing events and broadened our insight into how the disease alters gene expression in ways other than genomic alterations. In higher eukaryotes, the most common type of RNA editing is adenosine to inosine [21]. A previous study has reported that other types of RNA editing are prone to false-positives [22]. RNA editing events in coding regions can result in amino acid changes that affect the progression of cancer; therefore; we focused on A-to-G RNA editing sites in coding regions.

In this study, we focused on nonsynonymous RNA editing in CRC. First, we investigated RNA editing patterns by comparing 39 paired normal and tumor tissues. The number of 
RNA editing sites ranged from 12 to 42 according to our analysis. Consistent with previous studies, our results showed that there were few editing events in coding regions. We also investigated hyperediting and hypoediting ratios per sample and found that there were different patterns between patients, and no consistent change between cancerous and normal tissue.

Although we used relatively strict filtering standards, the possibility of false-positives remained owing to the potential for mis-mapping or sequencing errors. We removed this possibility by filtering with the IGV and BLAST programs, after which 10 positions in nine genes with nonsynonymous alterations were identified at the RNA level. Because we did not obtain tissue samples that were enrolled in the TCGA dataset, we only validated two editing sites, which were also detected in our RNA sequencing data of CRC cell lines. The DNA repair enzyme, NEIL1 transcript, which contains RNA editing, has distinct enzymatic properties [23]. RNA editing of SRP9, which was first identified in breast cancer [24], was also identified in the present study. We validated these positions using Sanger sequencing and the ICE method. Furthermore, using siRNA, we found that the ADAR enzyme affects these editing sites.

We investigated normal- or tumor-specific RNA editing sites. Among the recurrently detected sites, four were edited less frequently in cancer. One of these is IGFBP7, which has been studied as a tumor suppressor gene in a variety of cancers [25,26]. Edited IGFBP7 transcript, which has editing sites A284G and $A 232 G$, is less prone to cleavage, indicating that hypoedited IGFBP7 transcript in cancer is likely to be cleaved, possibly affecting cancer progression. Hypoediting of COPA (I164V) has also been found in hepatocellular carcinoma [27], but the effects of editing have yet to be elucidated.

We also found that editing of GLI1 was increased in CRC. To validate this result, we investigated the level of RNA editing of GLI1 from normal and tumor tissue samples using pyrosequencing. These results, from an independent cohort, demonstrate that GLI1 was overedited in CRC. GLI1 editing is also found in medulloblastoma, but the extent of editing is decreased in the tumor [28]. These opposing result suggests an interesting hypothesis; in the medulloblastoma, Hedgehog (Hh) signaling, which involves GLI1, promotes cell survival [29]. However, GLI1, a transactivator of Hh signaling, inhibits cell proliferation in CRC cells [30]. Although identical editing events were generated, they could have different roles according to various tissue types.

We analyzed nonsynonymous RNA editing in CRC and found hypoediting or hyperediting sites. These results provide a comprehensive view of the editing pattern in CRC. RNA editing of GLI1 was found to be increased, suggesting that it could be a novel biomarker for CRC. In addition, understanding the different RNA editing patterns of GLI1 from different tissues and tumor types will enhance our understanding of the role of RNA editing in cancer.

\section{Electronic Supplementary Material}

Supplementary materials are available at Cancer Research and Treatment website (http://www.e-crt.org).

\section{Conflicts of Interest}

Conflict of interest relevant to this article was not reported.

\section{Acknowledgments}

The authors are grateful to all members of our group for their helpful advice. This research was supported by a grant from the Korea Health Technology R\&D Project through the Korea Health Industry Development Institute, funded by the Ministry of Health \& Welfare (HI14C1277), by the Priority Research Centers Program through the NRF funded by the Ministry of Education, Science and Technology, Republic of Korea (2009-0093820), by the Doosan Yongkang Foundation (30-2013-015-0), and by the Ministry of Science, ICT \& Future Planning (2016M3A9B6026918).

\section{References}

1. Greenman C, Stephens P, Smith R, Dalgliesh GL, Hunter C, Bignell G, et al. Patterns of somatic mutation in human cancer genomes. Nature. 2007;446:153-8.

2. Cancer Genome Atlas Network. Comprehensive molecular characterization of human colon and rectal cancer. Nature. 2012;487:330-7.

3. Keegan LP, Gallo A, O'Connell MA. The many roles of an RNA editor. Nat Rev Genet. 2001;2:869-78.
4. Nishikura K. Editor meets silencer: crosstalk between RNA editing and RNA interference. Nat Rev Mol Cell Biol. 2006;7: 919-31.

5. Nishikura K. Functions and regulation of RNA editing by ADAR deaminases. Annu Rev Biochem. 2010;79:321-49.

6. Ju YS, Kim JI, Kim S, Hong D, Park H, Shin JY, et al. Extensive genomic and transcriptional diversity identified through massively parallel DNA and RNA sequencing of eighteen Korean 
individuals. Nat Genet. 2011;43:745-52.

7. Li M, Wang IX, Li Y, Bruzel A, Richards AL, Toung JM, et al. Widespread RNA and DNA sequence differences in the human transcriptome. Science. 2011;333:53-8.

8. Ramaswami G, Li JB. RADAR: a rigorously annotated database of A-to-I RNA editing. Nucleic Acids Res. 2014;42: D109-13.

9. Kiran A, Baranov PV. DARNED: a DAtabase of RNa EDiting in humans. Bioinformatics. 2010;26:1772-6.

10. Kleinman CL, Adoue V, Majewski J. RNA editing of protein sequences: a rare event in human transcriptomes. RNA. 2012;18:1586-96.

11. Paz N, Levanon EY, Amariglio N, Heimberger AB, Ram Z, Constantini $S$, et al. Altered adenosine-to-inosine RNA editing in human cancer. Genome Res. 2007;17:1586-95.

12. Cenci C, Barzotti R, Galeano F, Corbelli S, Rota R, Massimi L, et al. Down-regulation of RNA editing in pediatric astrocytomas: ADAR2 editing activity inhibits cell migration and proliferation. J Biol Chem. 2008;283:7251-60.

13. Maas S, Patt S, Schrey M, Rich A. Underediting of glutamate receptor GluR-B mRNA in malignant gliomas. Proc Natl Acad Sci U S A. 2001;98:14687-92.

14. Chen L, Li Y, Lin CH, Chan TH, Chow RK, Song Y, et al. Recoding RNA editing of AZIN1 predisposes to hepatocellular carcinoma. Nat Med. 2013;19:209-16.

15. Han SW, Kim HP, Shin JY, Jeong EG, Lee WC, Kim KY, et al. RNA editing in RHOQ promotes invasion potential in colorectal cancer. J Exp Med. 2014;211:613-21.

16. Koboldt DC, Zhang Q, Larson DE, Shen D, McLellan MD, Lin L, et al. VarScan 2: somatic mutation and copy number alteration discovery in cancer by exome sequencing. Genome Res. 2012;22:568-76.

17. Wang K, Li M, Hakonarson H. ANNOVAR: functional annotation of genetic variants from high-throughput sequencing data. Nucleic Acids Res. 2010;38:e164.

18. Sakurai M, Yano T, Kawabata H, Ueda H, Suzuki T. Inosine cyanoethylation identifies A-to-I RNA editing sites in the human transcriptome. Nat Chem Biol. 2010;6:733-40.
19. Oh JH, Ku JL, Yoon KA, Kwon HJ, Kim WH, Park HS, et al. Establishment and characterization of 12 human colorectalcarcinoma cell lines. Int J Cancer. 1999;81:902-10.

20. Han L, Diao L, Yu S, Xu X, Li J, Zhang R, et al. The genomic landscape and clinical relevance of A-to-I RNA editing in human cancers. Cancer Cell. 2015;28:515-28.

21. Maas S, Rich A, Nishikura K. A-to-I RNA editing: recent news and residual mysteries. J Biol Chem. 2003;278:1391-4.

22. Piskol R, Peng Z, Wang J, Li JB. Lack of evidence for existence of noncanonical RNA editing. Nat Biotechnol. 2013;31:19-20.

23. Yeo J, Goodman RA, Schirle NT, David SS, Beal PA. RNA editing changes the lesion specificity for the DNA repair enzyme NEIL1. Proc Natl Acad Sci U S A. 2010;107:20715-9.

24. Shah SP, Morin RD, Khattra J, Prentice L, Pugh T, Burleigh A, et al. Mutational evolution in a lobular breast tumour profiled at single nucleotide resolution. Nature. 2009;461:809-13.

25. Verhagen HJ, de Leeuw DC, Roemer MG, Denkers F, Pouwels W, Rutten A, et al. IGFBP7 induces apoptosis of acute myeloid leukemia cells and synergizes with chemotherapy in suppression of leukemia cell survival. Cell Death Dis. 2014;5:e1300.

26. Liu L, Yang Z, Zhang W, Yan B, Gu Q, Jiao J, et al. Decreased expression of IGFBP7 was a poor prognosis predictor for gastric cancer patients. Tumour Biol. 2014;35:8875-81.

27. Chan TH, Lin CH, Qi L, Fei J, Li Y, Yong KJ, et al. A disrupted RNA editing balance mediated by ADARs (Adenosine DeAminases that act on RNA) in human hepatocellular carcinoma. Gut. 2014;63:832-43.

28. Shimokawa T, Rahman MF, Tostar U, Sonkoly E, Stahle M, Pivarcsi A, et al. RNA editing of the GLI1 transcription factor modulates the output of Hedgehog signaling. RNA Biol. 2013;10:321-33.

29. Bar EE, Chaudhry A, Farah MH, Eberhart CG. Hedgehog signaling promotes medulloblastoma survival via Bc/II. Am J Pathol. 2007;170:347-55.

30. Akiyoshi T, Nakamura M, Koga K, Nakashima H, Yao T, Tsuneyoshi M, et al. Gli1, downregulated in colorectal cancers, inhibits proliferation of colon cancer cells involving Wnt signalling activation. Gut. 2006;55:991-9. 